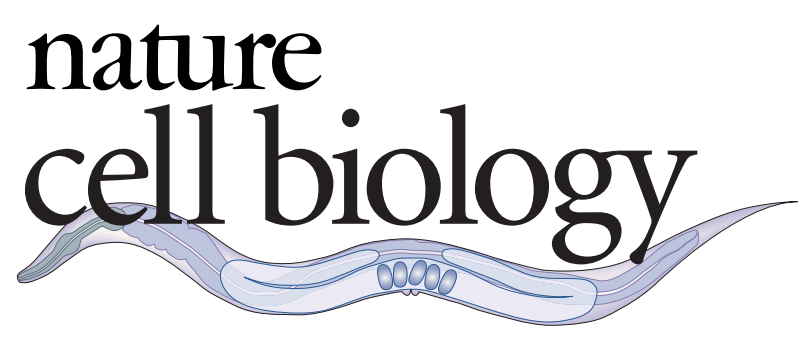

\title{
Nematodes net Nobel
}

R

obert Horvitz, John Sulston and Sydney Brenner are the recipients of this year's Nobel prize in Physiology or Medicine. The prize was awarded for development of the nematode worm Caenorhabditis elegans into what is now one of scientific community's favourite genetic models. Sydney Brenner (now president of the Molecular Sciences Institute, Berkeley) first happened upon the nematode in the 1960s when looking for a suitable system to study the development of the nervous system. He chose to study it over the more established fruit fly Drospohila melanogaster for its simplicity, transparency and short life cycle. As Brenner puts it, "we were trying to study behaviour genetically. [To achieve this, we split] the problem into two parts: one, how genes specify structure, and the other, how the nervous system generates behaviour."

In 1969, Sulston, until recently director of the Sanger Institute in Cambridge, joined Brenner's group in Cambridge. By the late 1970's, he had managed to document a complete lineage map of the 959 cells of the nematode adult purely on the basis of manual microscopic observation. It was a result of this remarkable achievement that apoptosis was observed in a developmental setting: 131 of the total 1090 cells would reproducibly die, evidently in a highly controlled manner. Robert Horvitz of the Massachusetts Institute of Technology was honoured for following in the 1980s, again in Brenner's lab, with the first characterization of genes regulating cell death, ced-3 and ced-4, in the nematode worm. Horvitz also noted the conservation of this pathway in higher eukaryotes, including humans.

Although this year's work is pegged on programmed cell death, after recent previous awards for studies of the cell cycle (yeast) and developmental biology (Drosophila melanogaster), this represents the third prize of recent times for fundamental work in a genetically tractable model organism.

Sulston has been a staunch proponent for free data access, especially in the context of the human genome project. He is keen to link this culture to the worm community, "In the worm community, we learnt how important public access was. From the beginning, this community was always celebrated for its co-operativeness: it helped us get stuff done".

For many years, Brenner had been widely tipped as a long overdue Nobel candidate, but more for his fundamental contributions to molecular biology than for honing in on the worm. John Sulston, on the other hand, has been in the limelight recently more as a key player in the sequencing of the worm genome and in the public human genome project, achievements that might well arouse the interest of a future Nobel committee. After all, the namesake of the institute he has headed until recently was honoured twice by the Swedish Academy.

\section{Nature Cell Biology's upwardly mobile impact factor: $\mathbf{2 1 . 9 4 4}$}

The 2001 impact factor for Nature Cell Biology has recently been revised by the Institute for Scientific Information (ISI), the institution behind the big number. This impact factor represents the average number of citations in 2001 to papers published in 1999 and 2000 (our first two years in print). After it was noticed that the denominator (that is, the total number of papers published) employed by ISI to calculate the impact factor for NCB was unaccountably large, ISI went back to crunch the numbers again. After recalculating, they arrived at a rating just short of 22 , more than 7 points above the value originally published (see erratum on http://isi6.isiknowledge.com; note that the ISI journal database itself has not been updated as of this month). 


\section{editorial}

Although we believe that the impact factor for a journal yields a sense of its overall standing, we would resist the temptation to use a simple numerical system such as this to rank journals covering different spectra of scientific research, and indeed different formats of publications. This exercise all too easily turns into a comparison of apples and oranges. We are keen to emphasize that although we follow our impact factor with great interest, it does not guide our editorial decisions. It is our hope, however, that our readers find the revised impact factor in line with the journal's recognized standing within the community it aims to serve.

\section{Microarray data standards}

This journal has always strived to ensure that the core data and materials of all the papers in our pages are accessible to every reader and sufficient to allow analysis and reproduction of the data. This represent a considerable challenge for rapidly evolving new technologies with complex data sets, such as that generated by microarray experiments. The problems are compounded by the multiple data formats available and the delayed evolution of broadly accepted public database depositories for such data.

Nature Cell Biology is now able to join the chorus of the other Nature journals by announcing that any paper presenting microarray data will be required to comply with the 'Minimal Information About a Microarray Experiment' (MIAME) standards for the publication of microarray data after December 1st 2002. We will ask for inclusion of the relevant accession numbers and that the data is posted in one of two public databases endorsed by the Micorarray and Gene Expression group (MGED) - GEO (www.ncbi.nlm.nih.gov/geo/)

and/or ArrayExpress (www.ebi.ac.uk/arrayexpress) - as a precondition for publication. We will also ask for submission of five compact disks containing microarray data in a format that complies with the checklist suggested by MGED (see http://www.mged.org/Workgroups/MIAME/miame_checklist.html).

This move aims to ensure that microarray data will be fully accessible to every reader and, crucially, refereed without barriers thrown up by proprietary software or incompatible data formats. This system will also ensure the anonymity of our referees. Further information will be posted on our guide to authors. We will continue to monitor the evolution of new technologies closely and adapt our publication strategies accordingly. As always, we welcome your feedback on this new policy. 